\title{
VIRTUELLES TREFFEN DER IAML-AUSTRIA UND DER VÖB-KOMMISSION FÜR MUSIK
}

\section{von Carl-Ulrich Friederici}

Zusammenfassung: Die VÖB-Kommission für Musik veranstaltete am 22.10.2020 gemeinsam mit der IAML-Austria ein virtuelles Treffen via Zoom. Es nahmen insgesamt 24 Personen teil, bestehend aus Mitgliedern beider Gruppen und einigen Gästen. Es waren damit 16 Institutionen vertreten. Das Treffen fand als Ersatz für die ausgefallene Präsenzsitzung Anfang Mai an der Fachbereichsbibliothek Musikwissenschaft der Universität Wien statt. Soweit sinnvoll und möglich, wurden die Themen der entfallenen Sitzung wieder aufgegriffen. Die Inhalte umfassten schwerpunktmäßig unterschiedliche Facetten der Informationsvermittlung und Informationsaufbereitung. Im Folgenden ein Bericht über den Verlauf.

Schlagwörter: VÖB-Kommission für Musik; IAML-Austria; virtuelles Treffen; Alma-Implementierung; RDA-Toolkit; 3R-Projekt; Katalogisierungshandbuch OBVSG; KEMÖ; Eigene Konsortien der Musikuniversitäten; Schreibzentrum Musikuniversität Wien

\section{VIRTUAL MEETING OF THE IAML-AUSTRIA AND THE VÖB COMMISSION FOR MUSIC}

Abstract: The VÖB Commission for Music organized a virtual meeting in cooperation with IAML-Austria on October 22 $2^{\text {nd }}, 2020$ via Zoom. A total of 24 people participated, consisting of members from both groups and a few guests. 16 institutions were represented. The meeting was a replacement for the canceled face-to-face meeting at the beginning of May at the library of the Musicology Department of the University of Vienna. As far as possible, the topics of the canceled meeting were readapted. The focus of the contents comprised different facets of information transfer and information processing. Below is a report on the process.

Keywords: VOEB-Commission for Music; IAML-Austria; virtual meeting; Alma implementation; RDA Toolkit; 3R-project; Cataloging Manual OBVSG; Austrian Academic Library Consortium; own consortia of music universities; The Writing Center of the University of Music Vienna

DOI: https://doi.org/10.31263/voebm.v73i3-4.5329

(c) Carl-Ulrich Friederici

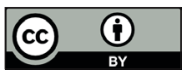

Dieses Werk ist - exkl. einzelner Logos und Abbildungen - lizenziert unter einer Creative-Commons-Lizenz Namensnennung 4.0 International-Lizenz 
Am 5. Mai 2020 sollte in der Fachbereichsbibliothek Musikwissenschaft der Universität Wien eine gemeinsame Sitzung der IAML-Austria und der VÖB-Kommission für Musik stattfinden. Diese Sitzung musste infolge des ersten Corona-Lockdowns ausfallen. Im Verlauf des Sommers war es dann absehbar, dass Präsenzsitzungen in diesem Jahr nicht stattfinden können. Zugleich wurde von Seiten des VÖB-Präsidiums darum gebeten, dass die Kommissionstätigkeiten nach Möglichkeit aufrechterhalten werden sollten.

Deshalb kamen die Vorsitzenden beider Gruppen überein eine virtuelle Sitzung gemeinsam durchzuführen, die auf ca. 2 Stunden begrenzt sein sollte. Mittels einer Doodle-Umfrage wurde der Termin auf den 22.10.2020 in der Zeit von 14.00-16.00 Uhr festgelegt.

$\mathrm{Da}$ an der Kunstuniversität Graz für virtuelle Meetings gerne die Plattform „Zoom" verwendet wird, entschied sich der Autor des Beitrags das Tool auch in diesem Fall zu nutzen. Das Meeting begann pünktlich ohne Probleme und alle 24 Teilnehmer*innen aus 16 verschiedenen Institutionen konnten die ganze Zeit das Meeting verfolgen, gleichgültig ob aus dem Büro oder aus dem Homeoffice.

Um dem Treffen zumindest eine gewisse persönliche Note zu geben, wurden die Teilnehmer*innen bei ihrem Eintritt einzeln begrüßt und das Wiedersehen im Allgemeinen als erfreuliches Ereignis gewürdigt. Die Einführung umfasste auch ein paar Anmerkungen zur Anwendung von Zoom während des Meetings. So sollte das Mikrofon nur während eines eigenen Wortbeitrags eingeschaltet sein. Der Wunsch danach sollte vorab durch ein Handzeichen signalisiert werden. Die Limitierung des kostenfreien Zoom-Accounts auf 40 Minuten wurde während der Video-Konferenz nicht wirksam, da zum betreffenden Zeitpunkt für Institutionen in Österreich diese Beschränkung nicht galt. Allerdings zeigte sich im weiteren Verlauf, dass einzelne Beitragende von Teilen des Auditoriums akustisch nur unzureichend beziehungsweise fast gar nicht zu verstehen waren. Mit dem Einverständnis aller ist die Videokonferenz als Hilfsmittel für die Protokollabfassung aufgezeichnet worden.

Auf der Sitzungsagenda standen mehrere aktuelle musikbibliothekarische Themen. Im Folgenden ist der Verlauf des Treffens nur in geraffter Form beschrieben. Ausführlicher wird auf die Inhalte im Protokoll eingegangen, welches auf der Kommissionsseite der VÖB-Homepage zur allgemeinen Verfügung steht.

Zunächst ging es um die Aktivitäten und Interna der beiden Gruppen. 


\section{Aktuelle Entwicklungen bei der IAML International}

Stefan Engl gab einen kurzen Überblick über die Entwicklungen bei der „International Association of Music Libraries, Archives and Documentation Centers" (IAML). Er berichtete über den Ablauf des virtuellen internationalen IAML-Kongresses in diesem Jahr und über das jährliche Treffen der IAML-Deutschland, das in einer hybriden Form stattfand, und gab einen Überblick über die weiteren geplanten internationalen Kongresse der kommenden Jahre. Die IAML-Austria wählt 2021 einen neuen Vorstand. Es wird um Bewerbungen für die zu besetzenden Posten gebeten. Das Fachblatt „Forum Musikbibliothek“ hat eine neue Schriftleitung. Für diese Zeitschrift sind Einreichungen von Beiträgen aus Österreich gewünscht.

\section{Interna der Kommission}

Die Interna der Kommission umfassten in der Hauptsache einige Personalia sowie die Genehmigung des letzten Sitzungsprotokolls.

\section{Alma-Implementierung}

Erstes größeres Thema der Zusammenkunft war die Implementierung von „Alma“, da einige Bibliotheken der österreichischen Musikuniversitäten Teil der Wave 7 sind. Aus diesem Anlass berichteten die Kolleg*innen der UB-Mozarteum Salzburg (Barbara Schwarz-Raminger und Daniel Revers) über den Verlauf und ihre bisher vorliegenden Erfahrungen bei der Migration der Daten nach „Alma“ und über die Präsentation in „Primo“, die kurz gezeigt wurde. Schwierigkeiten gab es vor allem in technischer Hinsicht und bei der Konfigurierung von „Primo“. Als größtes Problem beim Umstieg erwies sich aber die Implementierung des Ausleihsystems und der Nutzer*innenverwaltung. Ähnliche Probleme bestanden auch an der Musikuniversität Wien und an der Anton-Bruckner-Privatuniversität in Linz, wobei sich aber die Lösungen voneinander unterscheiden.

\section{3R-Projekt und Katalogisierungshandbuch OBVSG}

Thematisch passend zur Implementierung schloss sich ein Bericht von Christoph Steiger, UB der Musikuniversität Wien, zu Neuigkeiten zum 3R-Projekt an. Im Hinblick auf das zukünftige neue RDA-Toolkit soll ein 
RDA-Erschließungshandbuch für Bibliotheken im DACH-Raum erarbeitet werden, das in der Praxis als Ersatz zum Toolkit für die Katalogisierung zur Verfügung stehen soll.

Des Weiteren ging es in diesem Zusammenhang um die Einarbeitung von musikbezogenen Problemen in das OBVSG-Katalogisierungshandbuch, verbunden mit dem Aufruf an die Kolleg*innen, sich an dieser Arbeit zu beteiligen und sich bei bestehendem Interesse zu melden. Als erstes ist eine Anleitung zu GND-Ansetzungen von Musikwerken in „Alma“ geplant.

\section{KEMÖ und Musikkonsortien}

Das anschließende Thema beschäftigte sich mit dem elektronischen Informationsangebot in Musikbibliotheken. Robert Schiller, UB der Kunstuniversität Graz, trug einen durch eine PowerPoint-Präsentation unterstützten Beitrag zur „Kooperation E-Medien Österreich“ (KEMÖ) und zu Musikkonsortien vor, der eine Bestandsaufnahme der jetzigen Situation beinhaltete. Langfristig ist die Betreuung kleinerer Konsortien, zu denen viele musikspezifische Datenbanken zählen, durch die KEMÖ nicht gesichert. Einige werden schon jetzt gar nicht durch die Kooperation betreut. Es stellt sich die Frage, inwieweit die betroffenen Musikuniversitäten miteinander selbst Konsortien bilden sollten.

Ergänzend dazu ging der Beitrag auch auf das Steuerreformgesetz 2020 und die derzeitige nicht ganz geklärte Besteuerung von elektronischen Medien ein.

Das Konzept einer hybriden Bibliothek ist bis jetzt von den Musikbibliotheken in Österreich unterschiedlich stark umgesetzt worden. Eine Vorreiterrolle nimmt dabei die UB der Kunstuniversität Graz ein. Die folgenden Nachfragen zielten auf den Bedarf an elektronischen Ressourcen in Musikbibliotheken und auf die Abdeckung von entsprechenden Wünschen über die Musikwissenschaft hinaus.

\section{Aufbau des Schreibzentrums und die daraus entstandenen Services an der Musikuniversität Wien}

Eine besondere Initiative der Informationsvermittlung stellten im letzten Vortrag an diesem Tag Barbara Fuchslehner und Anna Eberhöfer von der UB der Musikuniversität Wien vor, zu dem es ebenfalls eine Power- 
Point-Präsentation gibt. Beim Schreibzentrum handelt es sich um ein Angebot an Studierende, das die UB seit dem Frühjahr 2020 als Hilfe zum Verfassen wissenschaftlicher Arbeiten anbietet. Bedingt durch die Einschränkungen ab März dieses Jahres wurden verstärkt E-Learning-Möglichkeiten für verschiedene Zielgruppen angeboten, unter anderem zu den Themen Literaturverwaltung und Zitieren. Weitere Angebote des Schreibzentrums auch für Betreuende sind geplant.

\section{Austausch zu aktuellen Themen}

Traditionellerweise wird während der Sitzungen der IAML-Austria und der Kommission für Musik der Informationsaustausch zu Aktualitäten aus den Institutionen gepflegt. Jede Bibliothek soll dabei über entsprechende Neuigkeiten berichten. Dieses ließ sich während dieser Zoom-Sitzung vor allem aus zeitlichen Gründen leider nicht im gewohnten Umfang umsetzen. Trotzdem konnte auf einige Wortmeldungen noch eingegangen werden. Es wurde sowohl über inhaltliche Projekte, wie zum Beispiel über die Gründung eines eigenen Verlags an der Musikuniversität Wien, als auch über personelle oder bauliche Veränderungen, wie zum Beispiel an der Kunstuniversität Graz, berichtet.

\section{Resümee}

Das Feedback zum abgehaltenen Meeting war durchwegs positiv und es stellte einen zweckmäßigen Ersatz für eine nicht mögliche Präsenzsitzung dar. Von den oben erwähnten akustischen Problemen abgesehen, gab es keine besonderen Schwierigkeiten. Allerdings ließ sich nach einigen Beiträgen eine gewisse Zurückhaltung spüren, auf die vermittelten Inhalte näher einzugehen.

Teilweise war dieses Treffen sogar im Ablauf konzentrierter und stringenter als manche Sitzung in traditioneller Form. Die einzelnen Themen ließen sich auch durch die Unterstützung von Präsentationen gut vermitteln. Die Zusammenkunft lässt sich also als erfolgreich bewerten und einige der Inhalte werden in Zukunft mit Sicherheit wieder aufgegriffen.

Es fehlt bei einer virtuellen Konferenz natürlich eine Art „Genius loci“; der persönliche Informationsaustausch zwischendurch und die Eindrücke, die man ansonsten aus der gastgebenden Bibliothek mitnehmen kann, entfallen leider. 
Unabhängig davon, ob die kommenden Treffen wieder in bisher üblicher Weise stattfinden oder noch weiterhin in virtueller Form abgehalten werden müssen, wird die Kommission für Musik ihre Arbeit in geeigneter und sinnvoller Art fortsetzen können.

Carl-Ulrich Friederici Universität für Musik und darstellende Kunst Graz, Universitätsbibliothek E-Mail: carl-ulrich.friederici@kug.ac.at 